\title{
The Convergence Rate of High-Dimensional Sample Quantiles for $\varphi$-Mixing Observation Sequences
}

\author{
Ling Peng * ${ }^{(1)}$ and Dong Han \\ School of Mathematical Sciences, Shanghai Jiao Tong University, Shanghai 200240, China; donghan@sjtu.edu.cn \\ * Correspondence: astridream@sjtu.edu.cn
}

\begin{abstract}
In this paper, we obtain the convergence rate for the high-dimensional sample quantiles with the $\varphi$-mixing dependent sequence. The resulting convergence rate is shown to be faster than that obtained by the Hoeffding-type inequalities. Moreover, the convergence rate of the high-dimensional sample quantiles for the observation sequence taking discrete values is also provided.
\end{abstract}

Keywords: convergence rate; sample quantiles; $\varphi$-mixing dependent sequence

check for updates

Citation: Ling, P.; Dong, H. The Convergence Rate of High-

Dimensional Sample Quantiles for $\varphi$-Mixing Dependent Observation Sequences. Mathematics 2021, 9, 647. https: / / doi.org/10.3390/math9060647

Received: 25 February 2021

Accepted: 17 March 2021

Published: 18 March 2021

Publisher's Note: MDPI stays neutral with regard to jurisdictional claims in published maps and institutional affiliations.

Copyright: (c) 2021 by the authors. Licensee MDPI, Basel, Switzerland. This article is an open access article distributed under the terms and conditions of the Creative Commons Attribution (CC BY) license (https:// creativecommons.org/licenses/by/ $4.0 /)$.

\section{Introduction}

In this paper, a high-dimensional observation sequence, $\mathbf{X}_{k}=\left(X_{1 k}, X_{2 k}, \ldots, X_{L k}\right)$, $1 \leq k \leq n$, is considered, where the dimension $L$ can be much larger than the sample size $n$. In particular, when the observation sequence comes from financial time series, the number of financial assets under observation can be much greater than the observation time. For instance, when considering $L$ stocks over a sequence of time periods $1 \leq k \leq n$, we denote $\mathbf{X}_{j k}$ as the stock return of the $j$-th assert over a period of time $k$, and its distribution characteristics are of our primary concern. Numerous empirical studies of the financial market indicated that the distributions of the stock returns $\mathbf{X}_{j k}$ were typically heavy-tailed, thus three continuous distributions with infinite mean: Cauchy, Levy and Pareto, are often used to model the tails in the returns ([1-3]). In the case of extremely heavy tails, it is known that the mean, $\mathbf{E}\left|X_{j k}\right|$, is infinite. Therefore, the sample median, or more generally, the sample quantile instead of the sample mean is often used to estimate the average location of $X_{j k}$. In those cases, quantile based methods play an important role in the modeling financial time series. For instance, Ando et al. [4] investigated the quantile co-movement of a huge number of financial time series based on panel data model, which captured the unobservable heterogeneity of the financial time series. Sim [5] estimated the correlation associated with the quantiles of asset returns, which implies the asset's dependence structure. Another application of the quantile in finance is Value-at-risk (VaR), which is a widely used measure of market risk associated with financial assets $([6,7])$. Given a positive value $q$, let $F(x)$ be the marginal distribution of the asset's returns, then the $1-q$ level VaR is defined as $\inf \{x: F(x) \geq q\}$, which is essentially a quantile. Therefore, the study of sample quantile estimator is of special importance in practical applications.

In order to study the behavior of the whole stock market, we may consider estimating the $q$-quantile of all risky assets simultaneously by the sample quantile estimators. In particular, our primary concern is the convergence rate of estimators. It is well known that the concentration inequality provides a measure of convergence rate. Given this, in this paper, we propose the concentration inequalities of the high-dimensional sample quantile estimators under the weak dependence conditions of $\varphi$-mixing, which cover a wide range of time series models. Recently, Howard and Ramdas [8] considered the problem of sequentially estimating quantiles of i.i.d. observations for a single variable, and they established a non-asymptotic and distribution-free concentration inequality for the empirical distribution function, which held uniformly over time with the law of the iterated logarithm. In addition to i.i.d. observations, there have been some works in recent years on sample 
quantiles under weak dependence condition. To name a few, Yang et al. [9] obtained the Berry-Esséen bound of the sample quantiles for $\varphi$ random variables with the rate of order $O\left(n^{-1 / 9}\right)$ under the condition of the mixing coefficient $\varphi(n)=O\left(n^{-\beta}\right)$ with $\beta \geq 13 / 6$. Yang et al. [10] further improved the rate of order to $O\left(n^{-1 / 6} \cdot \log n \cdot \log \log n\right)$ with mixing coefficients satisfying $\sum_{n=1}^{\infty} \varphi^{1 / 2}(n)<\infty$. Besides, Yang et al. [11] investigated the Bahadur representation for sample quantiles under the $\varphi$-mixing sequence with $\phi(n)=O\left(n^{-3}\right)$ and $\sum_{n=1}^{\infty} \varphi^{1 / 2}(n)<\infty$ respectively. For more recent works concerning the $\varphi$-mixing sequence, one can refer to [12-14]. However, in this paper, the concentration inequalities for the high-dimensional sample quantiles for the $\varphi$-mixing sequence are obtained without the assumption $\sum_{n=1}^{\infty} \varphi^{1 / 2}(n)<\infty$ on the mixing coefficients. Furthermore, based on the special property of the sample quantile, the convergence rate obtained in this paper is faster than that obtained by using the Hoeffding-type inequalities for the $\varphi$-mixing dependent sequence given in Rio [15] and Wang et al. [16] (see Remark 1).

The organization of this paper is as follows. The main results are presented in Section 2. Some preliminary lemmas are given in Section 3 and the proofs of the main theorem are provided in Section 4. Besides, a corollary is also proposed as an application of the main theorem.

\section{Main Results}

For each $j$, assume that $\left\{X_{j k}, k \geq 1\right\}$ is a sequence of random variables with a common marginal distribution function $F_{j}(x)$ and satisfy the following $\varphi$-mixing condition

$$
\begin{aligned}
\varphi_{j}(m) & =\max _{k \geq 1, A \in \mathfrak{F}(j)_{1}^{k}, \mathbf{P}^{(j)}(A)>0, B \in \mathfrak{F}(j)_{k+m}^{\infty}}\left|\mathbf{P}^{(j)}(B \mid A)-\mathbf{P}^{(j)}(B)\right| \\
\varphi_{j} & =\sum_{m=1}^{\infty} \varphi_{j}(m)<\infty
\end{aligned}
$$

where $\varphi_{j}(m)$ is decreasing on $m, \mathbf{P}^{(j)}(\cdot)$ denotes the probability distribution for the sequence $\left\{X_{j k}, k \geq 0\right\}$ and $\mathfrak{F}(j)_{k}^{m}=\sigma\left(X_{j i}, k \leq i \leq m\right)$ for $m \geq k \geq 1$.

For $0<q<1$, the $q$-th quantile $x_{j}(q)$ of $\left\{X_{j k}, k \geq 1\right\}$ for each $j$ is defined as

$$
x_{j}(q)=\inf \left\{x: F_{j}(x) \geq q\right\}
$$

For a sample $\left\{X_{j k}\right\}_{k=1}^{n}$, let $\hat{F}_{j n}$ represent the empirical distribution function based on $X_{j 1}, X_{j, 2}, \ldots, X_{j n}$, which is defined as $\hat{F}_{j n}(x)=n^{-1} \sum_{k=1}^{n} I\left(X_{j k} \leq x\right)$ for $x \in R$, where $I(\cdot)$ denotes the indicator function. For $0<q<1$, the $q$-th sample quantile $\hat{x}_{j n}(q)$ is defined as

$$
\hat{x}_{j n}(q)=\inf \left\{x: \hat{F}_{j n}(x) \geq q\right\}
$$

The exponential convergence rate of the high-dimensional sample quantile vector $\hat{\mathbf{x}}_{n}(q)=\left(\hat{x}_{1 n}(q), \ldots, \hat{x}_{L n}(q)\right)$ for $n \geq 1$ with the quantile vector $\mathbf{x}(q)=\left(x_{1}(q), \ldots, x_{L}(q)\right)$ is obtained under the following vector norm

$$
\left\|\hat{\mathbf{x}}_{n}(q)-\mathbf{x}(q)\right\|=\frac{1}{L} \sum_{j=1}^{L}\left|\hat{x}_{j n}(q)-x_{j}(q)\right|,
$$

Moreover, the following assumptions are needed in order to obtain the convergence rate.

Assumption 1. For each $j$, let $X_{j k}, 1 \leq k \leq n$, be continuous random variables. Assume that $F_{j}(x)$ is the second order differentiable, $F_{j}^{\prime \prime}(x)$ is bounded in a neighborhood of $x_{j}(q)$ and

$$
0<F_{j}\left(x_{j}(q)\right)<1, \quad 0<F_{j}^{\prime}\left(x_{j}(q)\right)<\infty
$$

for $1 \leq j \leq L$. 
Assumption 2. For each $j$, let $X_{j k}, 1 \leq k \leq n$ be discrete random variables taking discrete values $\left\{x_{m}^{j}\right\}$ and satisfying $x_{m}^{j}<x_{m+1}^{j}$, where $x_{m}^{j} \in S$. Assume that for some $0<q<1$, there is $i_{q}$ such that

$$
0<F_{j}\left(x_{i_{q}}^{j}\right)<q<F_{j}\left(x_{i_{q}+1}^{j}\right)<1,
$$

for $1 \leq j \leq L$, where $i_{q}$ may depends on $j$.

Now the following theorem gives the exponential convergence rate of the sample quantile vector $\hat{\mathbf{x}}_{n}(q)$.

Theorem 1. Let $0<q<1,\left\{X_{j k}, 1 \leq k \leq n\right\}$ have a common marginal distribution function $F_{j}(x)$ and satisfy the $\varphi$-mixing conditions (1) and (2) for $1 \leq j \leq L$. Let $n$ be large and $\log L / n$ be small.

(i) Let the Assumption 1 hold for $1 \leq j \leq L$. If $F_{q}^{\prime} \triangleq \min _{j \geq 1}\left\{F_{j}^{\prime}\left(x_{j}(q)\right)\right\}>0$ and $b_{q} \triangleq$ $\max _{j \geq 1}\left\{B_{j}\left(x_{j}(q)\right)\right\}<\infty$, then

$$
\boldsymbol{P}\left(\left\|\hat{\boldsymbol{x}}_{n}(q)-\boldsymbol{x}(q)\right\| \geq \frac{\delta_{n}}{F_{q}^{\prime}}\right) \leq 2(1+o(1)) \exp \left\{-\frac{n \delta_{n}^{2}}{\widetilde{b}_{q}}(1+o(1))\right\},
$$

for small $\delta_{n} \leq O(\log L / n)$, where

$$
B_{j}(x)=2\left(1+4 \varphi_{j}\right) F_{j}(x)\left(1-F_{j}(x)\right) .
$$

(ii) Let the Assumption 2 hold for $1 \leq j \leq$ L. If $m_{q} \triangleq \min _{j \geq 1}\left\{x_{i_{q}+1}^{j}-x_{i_{q}}^{j}\right\}>0$ and $c_{q} \triangleq$ $\min _{1 \leq j \leq L}\left\{C_{j}(q)\right\}>0$, then, for any positive number $M>1$ and small $M \sqrt{\log L / n}$,

$$
\boldsymbol{P}\left(\left\|\hat{\boldsymbol{x}}_{n}(q)-\boldsymbol{x}(q)\right\| \geq \varepsilon_{n}\right) \leq 2\left(1+\frac{2}{M}\right) L \exp \left\{-M c_{q} \sqrt{2 n \log L}(1+o(1))\right\}
$$

for any small positive numbers $\varepsilon_{n}$ satisfying $\varepsilon_{n}<m_{q}$, where

$$
C_{j}(q)=\min \left\{F_{j}\left(x_{i_{q}+1}^{j}\right)-q, \quad q-F_{j}\left(x_{i_{q}}^{j}\right)\right\}
$$

for $1 \leq j \leq L$

\section{Preliminary Lemmas}

Before proving the Theorem 1, we first prove two lemmas in the following. To keep the notations simple, we will omit the subscript $j$ in the following two lemmas.

Lemma 1. We denote $Y_{i}(x)=I\left(X_{i} \leq x\right)$ and $\bar{Y}_{i}(x)=Y_{i}(x)-F(x)$ for $i \geq 1$. Then

$$
\boldsymbol{E}\left(\sum_{i=k+1}^{k+m} \bar{Y}_{i}(x)\right)^{2} \leq m(1+4 \varphi) F(x)(1-F(x))
$$

for every $k \geq 1$ and $m \geq 1$.

Proof. It can easily be seen that $\left|\bar{Y}_{i}(x)\right| \leq 1$ with $\mathbf{E}\left(\bar{Y}_{i}(x)\right)=0, \mathbf{E}\left(\bar{Y}_{i}(x)\right)^{2}=F(x)(1-F(x))$ and $\mathbf{E}\left(\left|\bar{Y}_{i}(x)\right|\right)=2 F(x)(1-F(x))$. Applying the $\varphi$-mixing condition (1) we find that

$$
\begin{aligned}
\mathbf{E}\left(\bar{Y}_{i}(x) \bar{Y}_{l}(x)\right) & \leq \mathbf{E}\left(\left|\bar{Y}_{i}(x)\right| \mathbf{E}\left(\bar{Y}_{l}(x) \mid \mathfrak{F}_{0}^{i}\right)\right) \\
& \leq \varphi(l-i) \mathbf{E}\left(\left|\bar{Y}_{i}(x)\right|\right) \\
& =2 \varphi(l-i) F(x)(1-F(x))
\end{aligned}
$$


for $l>i$. Thus

$$
\begin{aligned}
\mathbf{E}\left(\sum_{i=k+1}^{k+m} \bar{Y}_{i}(x)\right)^{2} & \leq m F(x)(1-F(x))+4 F(x)(1-F(x)) \sum_{i=1}^{m-1} \sum_{l=i+1}^{m} \varphi(l-i) \\
& =m F(x)(1-F(x))\left(1+4 \sum_{i=1}^{m-1}\left(1-\frac{i}{m}\right) \varphi(i)\right) .
\end{aligned}
$$

This means that (5) holds.

Lemma 2. Assume that the condition in (2) holds. Then $n \varphi(n) \rightarrow 0$ as $n \rightarrow \infty$.

Proof. By using Kronecher's lemma, we have

$$
\varphi=\sum_{k=1}^{\infty} \frac{k \varphi(k)}{k}<\infty \quad \Longrightarrow \quad \frac{1}{n} \sum_{k=1}^{n} k \varphi(k) \rightarrow 0
$$

as $n \rightarrow \infty$.

Hence $n^{-1} \sum_{k=1}^{n} k \varphi(k) \geq \varphi(n) n^{-1} \sum_{k=1}^{n} k=(n+1) \varphi(n) / 2 \rightarrow 0$ as $n \rightarrow \infty$.

It can be seen that the two lemmas above hold for all $\varphi_{j}(),. 1 \leq j \leq L$, satisfying (2).

\section{Proofs of Main Results}

With the help of the preceding two lemmas, we are now turning to the proof of Theorem 1.

Proof of Theorem 1. (i). Note that

$$
\mathbf{P}\left(\left\|\hat{\mathbf{x}}_{n}(q)-\mathbf{x}(q)\right\| \geq \frac{\delta_{n}}{F_{q}^{\prime}}\right) \leq \sum_{j=1}^{L} \mathbf{P}\left(\left|\hat{x}_{j n}(q)-x_{j}(q)\right| \geq \frac{\delta_{n}}{F_{j}^{\prime}\left(x_{j}(q)\right)}\right) .
$$

It is clear that the inequality (3) holds as long as we can prove that for every $1 \leq j \leq L$,

$$
\mathbf{P}\left(\left|\hat{x}_{j n}(q)-x_{j}(q)\right| \geq \frac{\delta_{n}}{F_{j}^{\prime}\left(x_{j}(q)\right)}\right) \leq 2(1+o(1)) \exp \left\{-\frac{n \delta_{n}^{2}}{B_{j}\left(x_{j}(q)\right)}(1+o(1))\right\}
$$

for small $\delta_{n}$.

To keep the notations simple, we omit the subscript $j$ in the following discussion. Let $c_{q}=1 / F^{\prime}(x(q)), Y_{k}(x)=I\left(X_{k} \leq x\right)$ and $\bar{Y}_{k}(x)=Y_{k}(x)-F(x)$. Note that $F(x(q))=q$, it follows from the definition of the quantile and the Lagrange's mean value theorem that

$$
\begin{aligned}
\mathbf{P}\left(\hat{x}_{n}(q) \leq x(q)-c_{q} \delta_{n}\right) & \leq \mathbf{P}\left(q \leq \hat{F}_{n}\left(x(q)-c_{q} \delta_{n}\right)\right) \\
& =\mathbf{P}\left(\sum_{k=1}^{n} \bar{Y}_{k}\left(x(q)-c_{q} \delta_{n}\right) \geq n\left[F(x(q))-F\left(x(q)-c_{q} \delta_{n}\right)\right]\right) \\
& =\mathbf{P}\left(\sum_{k=1}^{n} \bar{Y}_{k}\left(x(q)-c_{q} \delta_{n}\right) \geq n\left[F^{\prime}(x(q)) c_{q} \delta_{n}+o(1)\right]\right) \\
& =\mathbf{P}\left(\sum_{k=1}^{n} \bar{Y}_{k}\left(x(q)-c_{q} \delta_{n}\right) \geq n \delta_{n}(1+o(1))\right) .
\end{aligned}
$$

We denote $S_{n}=\sum_{k=1}^{n} \bar{Y}_{k}\left(x(q)-c_{q} \delta_{n}\right)$. As in Ibragimov [17] and Yang [18], the sum $S_{n}$ can be written as two parts

$$
S_{n}=\sum_{i=1}^{l+1} \xi_{i}+\sum_{i=1}^{l} \eta_{i}
$$


where

$$
\xi_{i}=\sum_{j=1}^{m} \bar{Y}_{2(i-1) m+j}\left(x(q)-c_{q} \delta_{n}\right), \quad \eta_{i}=\sum_{j=1}^{m} \bar{Y}_{(2 i-1) m+j}\left(x(q)-c_{q} \delta_{n}\right),
$$

for $1 \leq i \leq l$, and

$$
\xi_{l+1}=\left\{\begin{array}{lll}
0 & \text { for } & 2 l m \geq n, \\
\bar{Y}_{2 l m+1}\left(x(q)-c_{q} \delta_{n}\right)+\ldots+\bar{Y}_{n}\left(x(q)-c_{q} \delta_{n}\right) & \text { for } & 2 l m<n,
\end{array}\right.
$$

where we let $\bar{Y}_{n+1}\left(x(q)-c_{q} \delta_{n}\right), \bar{Y}_{n+2}\left(x(q)-c_{q} \delta_{n}\right), \ldots, \bar{Y}_{2 l m}\left(x(q)-c_{q} \delta_{n}\right)$ in the above terms all equal 0 when $2 \mathrm{~lm}>n$.

Moreover, let

$$
m=\left\lfloor\sqrt{\frac{n}{2 M^{4} \log L}}\right\rfloor+1 \quad \text { and } \quad l=\left\lfloor\sqrt{\frac{n M^{4} \log L}{2}}\right\rfloor,
$$

where $\lfloor x\rfloor$ denotes the maximum integer less or equal to $x$, and $M$ is a positive number satisfying that for any given small $\epsilon(1>\epsilon>0), 1 / M \leq \epsilon$. It is clear that $2 l(m-1) \leq n<$ $2(l+1) m$ and $2 l(m-1) / n \rightarrow 1$ as $n \rightarrow \infty$.

According to (7), by the Chebyshev's inequality and Hölder's inequality, we have

$$
\begin{aligned}
& \mathbf{P}\left(\hat{x}_{n}(q)<x(q)-c_{q} \delta_{n}\right) \\
\leq & \mathbf{P}\left(\sum_{k=1}^{n} \bar{Y}_{k}\left(x(q)-c_{q} \delta_{n}\right) \geq n \delta_{n}(1+o(1))\right) \\
\leq & \exp \left\{-\theta n \delta_{n}(1+o(1))\right\} \operatorname{E} \exp \left\{\theta S_{n}\right\} \\
\leq & \exp \left\{-\theta n \delta_{n}(1+o(1))\right\}\left(\operatorname{E} \exp \left\{2 \theta \sum_{i=1}^{l+1} \xi_{i}\right\}\right)^{1 / 2}\left(\operatorname{E} \exp \left\{2 \theta \sum_{i=1}^{l} \eta_{i}\right\}\right)^{1 / 2}
\end{aligned}
$$

for $\theta>0$. Note that $\left|\bar{Y}_{i}(x)\right| \leq 1$, by the definition of $\xi_{i}$ in (8), we have $\left|\xi_{i}\right| \leq m$. Let $\theta \leq M \sqrt{2 \log L / n}$, recalling the definition of $m$ in (9), we have

$$
2 \theta\left|\xi_{i}\right| \leq 2 \theta m \leq 1 / M \leq \epsilon
$$

for $1 \leq i \leq l+1$. Then if follows from (10) that

$$
\begin{aligned}
& \mathbf{P}\left(\hat{x}_{n}(q)<x(q)-c_{q} \delta_{n}\right) \\
\leq & e^{\epsilon} \exp \left\{-\theta n \delta_{n}(1+o(1))\right\}\left(\mathbf{E} \exp \left\{2 \theta \sum_{i=1}^{l} \xi_{i}\right\}\right)^{1 / 2}\left(\operatorname{E} \exp \left\{2 \theta \sum_{i=1}^{l} \eta_{i}\right\}\right)^{1 / 2}
\end{aligned}
$$

Note that $e^{\epsilon / 2} \leq 1+\epsilon$ for $\epsilon \leq 2$. It follows that

$$
\begin{aligned}
\log \mathbf{E}\left(\exp \left\{2 \theta \xi_{i}\right\}\right) & \leq \log \left(1+2 \theta^{2} \mathbf{E}\left(\xi_{i}^{2}\right)(1+\epsilon)\right) \\
& \leq 2 \theta^{2} \mathbf{E}\left(\tilde{\zeta}_{i}^{2}\right)(1+\epsilon)
\end{aligned}
$$

and therefore, applying the Lemma 1, we have

$$
\begin{aligned}
\mathbf{E}\left(\exp \left\{2 \theta \xi_{i}\right\}\right) & \leq \exp \left\{2 \theta^{2} \mathbf{E}\left(\tilde{\zeta}_{i}^{2}\right)(1+\epsilon)\right\} \\
& \leq \exp \left\{\theta^{2} m B(x(q))(1+o(1))(1+\epsilon)\right\} .
\end{aligned}
$$

Furthermore, by using the similar way of proving the Lemma 6 in Yang [18], it follows from (12) and the $\varphi$-mixing condition in (1) that 


$$
\begin{aligned}
\mathbf{E}\left(\exp \left\{2 \theta \sum_{i=1}^{l} \xi_{i}\right\}\right) & =\mathbf{E}\left(\exp \left\{2 \theta \sum_{i=1}^{l-1} \xi_{i}\right\} \mathbf{E}\left(\exp \left\{2 \theta \xi_{l}\right\} \mid \mathfrak{F}_{0}^{l-1}\right)\right) \\
& \leq\left(\mathbf{E}\left(\exp \left\{2 \theta \xi_{l}\right\}\right)+e^{\epsilon} \varphi(m)\right) \mathbf{E}\left(\exp \left\{2 \theta \sum_{i=1}^{l-1} \xi_{i}\right\}\right) \\
& \leq \exp \left\{e^{\epsilon} \varphi(m)\right\} \exp \left\{\theta^{2} m B(x(q))(1+o(1))(1+\epsilon)\right\} \mathbf{E}\left(\exp \left\{2 \theta \sum_{i=1}^{l-1} \xi_{i}\right\}\right) \\
& \leq \cdots \\
& \leq \exp \left\{e^{\epsilon} \varphi(m) l\right\} \exp \left\{\theta^{2} m l B(x(q))(1+o(1))(1+\epsilon)\right\} .
\end{aligned}
$$

Similarly, we can obtain that

$$
\mathbf{E}\left(\exp \left\{2 \theta \sum_{i=1}^{l} \eta_{i}\right\}\right) \leq \exp \left\{e^{\epsilon} \varphi(m) l\right\} \exp \left\{\theta^{2} m l B(x(q))(1+o(1))(1+\epsilon)\right\} .
$$

By the definitions of $m$ and $l$ in (9) we have $\varphi(m) l=M^{4} \varphi(m) m \log L$. Thus, combining (11), (13) with (14), it follows that

$$
\begin{aligned}
& \mathbf{P}\left(\hat{x}_{n}(q)<x(q)-c_{q} \delta_{n}\right) \\
\leq & e^{\epsilon} \exp \left\{e^{\epsilon} \varphi(m) l\right\} \exp \left\{-\theta n \delta_{n}(1+o(1))\right\} \exp \left\{\theta^{2} m l B(x(q))(1+o(1))(1+\epsilon)\right\} \\
= & e^{\epsilon} \exp \left\{e^{\epsilon} \varphi(m) l\right\} \exp \left\{-n\left[\delta_{n} \theta-(1+\epsilon) B(x(q)) \theta^{2} / 2\right](1+o(1))\right\} \\
= & e^{\epsilon} \exp \left\{-n\left(-e^{\epsilon} M^{4} \varphi(m) m \log L / n+\left[\delta_{n} \theta-(1+\epsilon) B(x(q)) \theta^{2} / 2\right](1+o(1))\right)\right\}
\end{aligned}
$$

for $\theta \leq M \sqrt{2 \log L / n}$. Since $\epsilon>0$ can be arbitrary small and, applying the lemma 2, $\varphi(m) m \rightarrow 0$ as $n \rightarrow \infty$ ( and therefore, $m \rightarrow \infty$ ), it follows that

$$
\mathbf{P}\left(\hat{x}_{n}(q)<x(q)-c_{q} \delta_{n}\right) \leq(1+o(1)) \exp \left\{-\frac{n \delta_{n}^{2}}{B(x(q))}(1+o(1))\right\} .
$$

for large $n$. Similarly, we can obtain that

$$
\mathbf{P}\left(\hat{x}_{n}(q)>x(q)+c_{q} \delta_{n}\right) \leq(1+o(1)) \exp \left\{-\frac{n \delta_{n}^{2}}{B(x(q))}(1+o(1))\right\} .
$$

for large $n$. The two inequalities (16) and (17) mean that the inequality (6) holds for $1 \leq j \leq L$ and therefore, the concentration inequality (3) is true.

(ii). Let $F\left(x_{i_{q}}\right)<q<F\left(x_{i_{q}+1}\right)$. Since $x(q)=x_{i_{q}+1}$ and $F\left(x(q)-\varepsilon_{n}\right)=F\left(x_{i_{q}+1}-\varepsilon_{n}\right)=$ $F\left(x_{i_{q}}\right)$ for $\varepsilon_{n}$ satisfying $\varepsilon_{n}<x_{i_{q}+1}-x_{i_{q}}$, it follows that

$$
\begin{aligned}
\mathbf{P}\left(\hat{x}_{n}(q) \geq x(q)\right) & \leq \mathbf{P}\left(q \geq \hat{F}_{n}(x(q))\right) \\
& =\mathbf{P}_{1}\left(-\sum_{k=1}^{n} \bar{Y}_{k}(x(q)) \geq n\left(F\left(x_{i_{q}+1}\right)-q\right)\right), \\
\mathbf{P}\left(-\varepsilon_{n} \geq \hat{x}_{n}(q)-x(q)\right) & \leq \mathbf{P}\left(q \leq \hat{F}_{n}\left(x(q)-\varepsilon_{n}\right)\right) \\
& =\mathbf{P}\left(\sum_{k=1}^{n} \bar{Y}_{k}\left(x(q)-\varepsilon_{n}\right) \geq n\left(q-F\left(x_{i_{q}}\right)\right)\right) .
\end{aligned}
$$


Similar to (15), taking $\theta=M \sqrt{2 \log L / n}$ we can obtain that

$$
\begin{aligned}
& \mathbf{P}\left(\hat{x}_{n}(q) \geq x(q)\right) \\
\leq & e^{\epsilon} \exp \left\{-n\left(-e^{\epsilon} M^{4} \varphi(m) m \log L / n+\right.\right. \\
& {\left.\left.\left[\left(F\left(x_{i_{q}+1}\right)-q\right) \theta-(1+\epsilon) B(x(q)) \theta^{2} / 2\right](1+o(1))\right)\right\} } \\
\leq & \left(1+\frac{2}{M}\right) \exp \left\{-M\left(F\left(x_{i_{q}+1}\right)-q\right) \sqrt{2 n \log L}(1+o(1))\right\}
\end{aligned}
$$

for large $n$ and small $\sqrt{\log L / n}$. Similar to (18), we have

$$
\mathbf{P}\left(-\varepsilon_{n} \geq \hat{x}_{n}(q)-x(q)\right) \leq\left(1+\frac{2}{M}\right) \exp \left\{-M\left(q-F\left(x_{i_{q}}\right)\right) \sqrt{2 n \log L}(1+o(1))\right\}
$$

for $\varepsilon_{n}<m_{q}$, large $n$ and small $\sqrt{\log L / n}$. The two inequalities (18) and (19) combined imply that the inequality (4) is true. This completes the proof of Theorem 1.

Remark 1. Let $B_{j}(x)=B(x)$ for all $j$. Since $\left\|\bar{Y}_{j}\right\|_{\infty}^{2}=\max \left\{F^{2},(1-F)^{2}\right\}$ and $F(1-F) \leq$ $\max \left\{F^{2},(1-F)^{2}\right\}$, it follows that the coefficient $b_{q}=2(1+4 \varphi) F(1-F)$ in (i) is less than $2(1+4 \varphi) \max \left\{F^{2},(1-F)^{2}\right\}$ and therefore, the convergence rate in (i) is faster than that obtained by the Hoeffding-type inequality given in Corollary 2.1 by Rio [15]. Note that $\boldsymbol{E}\left(\bar{Y}_{j}^{2}\right)=F(1-F)$. When $2(1+4 \varphi) \leq 32$, the convergence rate in (i) is faster than that obtained by the Hoeffding-type inequality given by Wang et al. [16] on a measurable set $S$ such that for small $\delta>0, \boldsymbol{P}(S) \geq 1-\delta$. Though Wang et al. [16] considered the unbounded $\varphi$-mixing dependent sequences, it needs to assume that $\sum_{m=1}^{\infty}(\varphi(m))^{1 / 2}<\infty$.

As an application of (i) of Theorem 1, we can get the following corollary.

Corollary 1. Assume that $0<q<1$ and the conditions in (i) of Theorem 1 hold. Let $\delta_{n}=$ $c \sqrt{\log L / n}$. If $c>\sqrt{2 b_{q}}$, then

$$
\left\|\hat{x}_{n}(q)-x(q)\right\|<\frac{c}{F_{q}^{\prime}} \sqrt{\frac{\log L}{n}}
$$

with the probability at least $1-2 / L$ for large $L$ and small $\log L / n$. In particular, if $\left\{X_{j k}, k \geq 0\right\}$ is i.i.d. for every $j$, then $\varphi_{j}=0, B_{j}\left(x_{j}(q)\right)=2 q(1-q)$ for every $j$ and therefore, $b_{q}=2 q(1-q)$.

\section{Discussion}

It can be seen that the concentration inequalities are obtained without the assumption $\sum_{n=1}^{\infty} \varphi^{1 / 2}(n)<\infty$ on the mixing coefficients. Besides, the convergence rate is faster than that obtained by Hoeffding-type inequality for $\varphi$-mixing observation sequences. Moreover, the convergence rate of sample quantiles for the observation sequence taking discrete values is also given.

If the assumption that there is some correlation between the $L$ sequences $\left(X_{1 k}: k \geq\right.$ $0), \ldots,\left(X_{L k}: k \geq 0\right)$ is made, maybe one can obtain a better convergence rate. It is a problem worthy of further study.

Author Contributions: Conceptualization, L.P. and D.H.; methodology, D.H.; formal analysis, L.P. and D.H.; writing—original draft preparation, L.P. and D.H.; writing—review and editing, L.P. and D.H.; supervision, D.H.; project administration, D.H. All authors have read and agreed to the published version of the manuscript.

Funding: This research was funded by National Natural Science Foundation of China (No. 11531001). 
Acknowledgments: The authors would like to thank the anonymous referees whose valuable comments and helpful suggestions have greatly improved this manuscript.

Conflicts of Interest: The authors declare no conflict of interest.

\section{References}

1. Bhattacharya, R.N.; Waymire, E.C. Stochastic Processes with Applications; John Wiley \& Sons Inc.: New York, NY, USA, 1990; p. $x v i+672$.

2. Jondeau, E.; Rockinger, M. Testing for differences in the tails of stock-market returns. J. Empir. Financ. 2003, 10, 559-581. [CrossRef]

3. Nolan, J.P. Modeling financial data with stable distributions. In Handbook of Heavy Tailed Distributions in Finance; Svetlozar, T.R., Ed.; Elsevier: Amsterdam, The Netherlands, 2003; p. 105-130.

4. Ando, T.; Bai, J. Quantile co-movement in financial markets: A panel quantile model with unobserved heterogeneity. J. Am. Stat. Assoc. 2020, 115, 266-279. [CrossRef]

5. Sim, N. Modeling the dependence structures of financial assets through the Copula Quantile-on-Quantile approach. Int. Rev. Financ. Anal. 2016, 48, 31-45. [CrossRef]

6. Ibragimov, R.; Prokhorov, A. Heavy tails and copulas: Limits of diversification revisited. Econom. Lett. 2016, 149, 102-107. [CrossRef]

7. McNeil, A.J.; Frey, R.; Embrechts, P. Quantitative Risk Management: Concepts, Techniques and Tools, Revised ed.; Princeton University Press: Princeton, NJ, USA, 2015; p. xix+699.

8. Howard, S.R.; Ramdas, A. Sequential estimation of quantiles with applications to a/b-testing and best-arm identification. arXiv 2019, arXiv:1906.09712.

9. Yang, W.Z.; Wang, X.J.; Li, X.Q.; Hu, S.H. Berry-esséen bound of sample quantiles for $\varphi$-mixing random variables. J. Math. Anal. Appl. 2012, 388, 451-462. [CrossRef]

10. Yang, W.Z.; Wang, X.J.; Hu, S.H. A Note on the Berry-Esséen bound of sample quantiles for $\phi$-mixing sequence. Comm. Statist. Theory Methods 2014, 43, 4187-4194. [CrossRef]

11. Yang, W.Z.; Hu, S.H.; Wang, X.J. The Bahadur representation for sample quantiles under dependent sequence. Acta Math. Appl. Sin. 2019, 35, 521-531. [CrossRef]

12. Ding, L.; Chen, P.; Li, Y. Statistical inference for a heteroscedastic regression model with $\varphi$-mixing errors. Commun. Stat. Simul. Comput. 2020, 1-19. [CrossRef]

13. Wang, W.J.; Hu, S.H.; Shen, Y.; Yang, W.Z. Moment inequality for $\varphi$-mixing sequences and its applications. J. Inequal. Appl. 2009, $2009,379743$.

14. Xi, M.; Wang, X. On the rates of asymptotic normality for recursive kernel density estimators under $\phi$-mixing assumptions. J. Nonparametr. Stat. 2019, 31, 340-363. [CrossRef]

15. Rio, E. Asymptotic Theory of Weakly Dependent Random Processes; Springer: Berlin, Germany, 2017; p. xviii+204.

16. Wang, X.J.; Wang, S.J.; Wang, R. Exponential inequalities for sums of unbounded $\varphi$-mixing sequence and their applications. Comm. Statist. Theory Methods 2017, 46, 457-464. [CrossRef]

17. Ibragimov, I.A. Some limit theorems for stationary processes. Theory Probab. Appl. 1962, 7, 349-382. [CrossRef]

18. Yang, S.C. Almost sure convergence of weighted sums of mixing sequences. J. Syst. Sci. Math. Sci. 1995, 15, 254-265. (In Chinese) 